\title{
A User Cooperative Beamforming Approach to PAPR Reduction in MIMO-OFDM Uplink
}

\author{
Antti Arvola*, Antti Tölli*, and David Gesbert ${ }^{\dagger}$ \\ ${ }^{*}$ Centre for Wireless Communications, University of Oulu, \\ P.O. Box 4500, 90014 University of Oulu, Finland \\ E-mail: \{antti.arvola, antti.tolli\}@ee.oulu.fi \\ ${ }^{\dagger}$ EURECOM, Campus SophiaTech, 450 Route des Chappes, 06410 Biot Sophia Antipolis, France \\ E-mail: david.gesbert@eurecom.fr
}

\begin{abstract}
We consider a scheme for PAPR reduction based on user cooperation in a single-cell multi-user MIMO-OFDM uplink system. The idea is to utilize the unused space-frequency resources induced by collaborative beamforming to transmit dummy symbols, resulting in a transmit signal with reduced perantenna peak powers. Traditionally collaborative beamforming has been used to acquire beamforming gain, i.e., extended cell coverage and improved rates, while our approach also in addition reduces the transmit signal PAPR of individual user antennas. The reduced PAPR enables the users to allocate more power to data transmission, resulting in a higher system sum-rate for a fixed peak transmit power budget while also relaxing the strict linearity requirements of the user equipment RF components. We also account for the power required to exchange data between cooperating users by utilizing a simple AWGN channel model between users. We formulate the PAPR minimization problem as a convex optimization problem that reduces the PAPR of a given time domain transmit signal provided there are subcarriers with no previous bit allocations, which is a valid assumption especially at mid- and low-SNR regimes.
\end{abstract}

\section{INTRODUCTION}

MIMO-OFDM has been established as a proficient technology in wireless communications, providing high data rates and robustness in frequency selective communication. A major drawback of OFDM, however, has been the high peak-toaverage power ratio (PAPR) that casts strict requirements for $\mathrm{RF}$ components in the user equipment and causes non-linear distortion in the transmit power amplifiers (PA) as the signal peaks reach the non-linear operating zone of the PA. Due to the distortion and the resulting spreading in the frequency domain, PAPR has to be accounted for by operating the amplifiers at the linear region, resulting in poor power efficiency. This back-off is directly linked to the transmit signal PAPR, and significant gains can be achieved by reducing the signal fluctuations. Thus, PAPR reduction in OFDM has been a research topic of significant interest, and a multitude of different methods have been devised, see [1]-[5] for example.

User cooperation can be exploited to provide robustness and reliability on the wireless transmission and simultaneously

This research was supported by the Academy of Finland (Decision no. 279101 and 284590). D. Gesbert is supported by the ERC under the European Unions Horizon 2020 research and innovation program (Agreement no. 670896). increase the transmission rates. Traditionally, the user cooperation is utilized to obtain beamforming gain while joint data transmission can also provide spatial multiplexing benefits for single antenna users. Some of the earlier work, such as [6], [7], utilize user diversity to increase transmission reliability. The cooperation between users can be established via deviceto-device (D2D) communication protocols that depend on the distance between users. A survey into various D2D methods is given in [8]. One aspect of our work is the ability to account for the inter-user distance in power budget optimization.

In this paper, we approach PAPR reduction in a multi-user uplink system by exploiting the orthogonality of different data streams and unused space-frequency resources (i.e., empty subcarriers). We consider user collaboration to achieve beamforming gain and an exploitable nullspace (with respect to data-carrying streams) and formulate an optimization problem to find the transmitted complex dummy symbols based on given time domain transmit waveform. The authors in [9] considered a downlink scenario and jointly optimized the transmit precoding, OFDM modulation and PAPR reduction with a large number of transmit antennas by minimizing the $l_{\infty}$-norm of the transmitted precoded signal. In this paper, we take a very similar but different approach. Our aim is to maximize the throughput of the multi-user uplink system under a constraint on the user peak transmit powers. We utilize the Hughes-Hartogs (HH) [10] bit and power allocation to obtain the transmit sequence, while [9] considered a constant alphabet. We account for the user cooperation power requirement by utilizing a simple additive white Gaussian noise (AWGN) channel model between users that can be used to calculate the additional power term. The acquisition of transmit and receive beamformers and the optimization of dummy symbols is considered separately, i.e., the PAPR minimization scheme can be applied to any possible beamformer and/or bit loading approach.

\section{System Model}

We consider a multi-user MIMO-OFDM multicarrier singlecell system in uplink with $N_{c}$ orthogonal narrowband subcarriers, where a base station (BS) with $M$ antennas serves $K$ single-antenna users (UE, user equipment) utilizing different 
levels of transmit cooperation. The levels of cooperation considered are full cooperation via collaborative transmit beamforming and data transmission, and no cooperation with separate user transmissions. The detected signal on subcarrier $c$ and stream $s$ is given by

$\hat{x}_{c, s}=\mathbf{u}_{c, s}^{\mathrm{H}} \mathbf{H}_{c} \mathbf{v}_{c, s} \sqrt{p_{c, s}} x_{c, s}+\mathbf{u}_{c, s}^{\mathrm{H}} \mathbf{H}_{c} \sum_{\substack{i=1 \\ i \neq s}}^{K} \mathbf{v}_{c, i} \sqrt{p_{c, i}} x_{c, i}+\mathbf{u}_{c, s}^{\mathrm{H}} \mathbf{n}_{c}$,

where $\mathbf{u}_{c, s} \in \mathbb{C}^{M \times 1}$ and $\mathbf{v}_{c, s} \in \mathbb{C}^{K \times 1}$ denote the receive and transmit beamformers, respectively, $\mathbf{H}_{c}=\left[\mathbf{h}_{c, 1} \mathbf{h}_{c, 2} \ldots \mathbf{h}_{c, K}\right]$ $\in \mathbb{C}^{M \times K}$ concatenates the channel vectors of different users, $p_{c, s}$ and $x_{c, s}$ denote the transmit power and transmitted symbol from a QAM modulation scheme with $\mathbb{E}\left[\left|x_{c, s}\right|^{2}\right]=1, \forall c, s$ and $\mathbf{n}_{c}$ denotes the white Gaussian noise vector with per-element variance $N_{0}$. In this paper, we utilize the Hughes-Hartogs bit and power allocation scheme to generate the transmit symbols. For HH to work, zero interference is required between the data streams. Due to this fact, the receive and transmit beamformers are acquired either from SVD in case of full cooperation and joint beamforming, or zero-forcing in case of no cooperation. With full cooperation, the channel matrix is decomposed as $\mathbf{H}_{c}=\mathbf{U}_{c} \Lambda_{c} \mathbf{V}_{c}^{\mathrm{H}}$, where $\mathbf{U}_{c}=\left[\begin{array}{lll}\mathbf{u}_{c, 1} & \mathbf{u}_{c, 2} \ldots \mathbf{u}_{c, M}\end{array}\right] \in \mathbb{C}^{M \times M}$ and $\mathbf{V}_{c}=\left[\begin{array}{llll}\mathbf{v}_{c, 1} & \mathbf{v}_{c, 2} & \ldots & \mathbf{v}_{c, K}\end{array}\right] \in \mathbb{C}^{K \times K}$ denote the receive and transmit beamformers on subcarrier $c$, respectively. With no cooperation, the transmit beamformer reduces to identity $\mathbf{V}_{c}=\mathbf{I}, \forall c$, and the receiver is the zero-forcing receiver as $\mathbf{U}_{c}=\left(\mathbf{H}_{c}^{\mathrm{H}} \mathbf{H}_{c}\right) \mathbf{H}_{c}^{\mathrm{H}}, \forall c$. We also assume that $M \geq K$.

The UE-BS channel model considered in this paper is the classical multipath model for uniform linear arrays given by [11]

$$
\mathbf{h}_{c, k}=\frac{l_{d}}{\sqrt{L}} \sum_{l=1}^{L} \mathbf{a}\left(\alpha_{k, l}\right) e^{j \phi_{k, l}}, \forall c,
$$

where $l_{d}$ is the path loss between the user and base station, $L$ denotes the number of independent (and identically distributed, i.i.d.) propagation paths and $\phi_{k, l}$ is a random phase noise caused by the signal path $l$. We assume that all the users are equidistant from the base station, with a normalized average path loss of $l_{d}=1$. The vector $\mathbf{a}\left(\alpha_{k, l}\right)$ is the array signature vector for the angle of arrival $\alpha_{k, l}$ given by

$$
\mathbf{a}(\alpha)=\left[\begin{array}{c}
1 \\
e^{-j 2 \pi \frac{D}{\lambda} \cos (\alpha)} \\
\vdots \\
e^{-j 2 \pi \frac{(M-1) D}{\lambda} \cos (\alpha)}
\end{array}\right],
$$

where $D$ is the antenna spacing and $\lambda$ is the carrier wavelength.

The channel between users is modeled as a simple point-topoint AWGN channel, which only depends on the UE-UE path loss. In addition to being equidistant from the base station, the users are assumed to have an angle between their respective angles of arrival, denoted with $\theta$. The user distance from the base station is denoted with $d$, while the inter-user distance

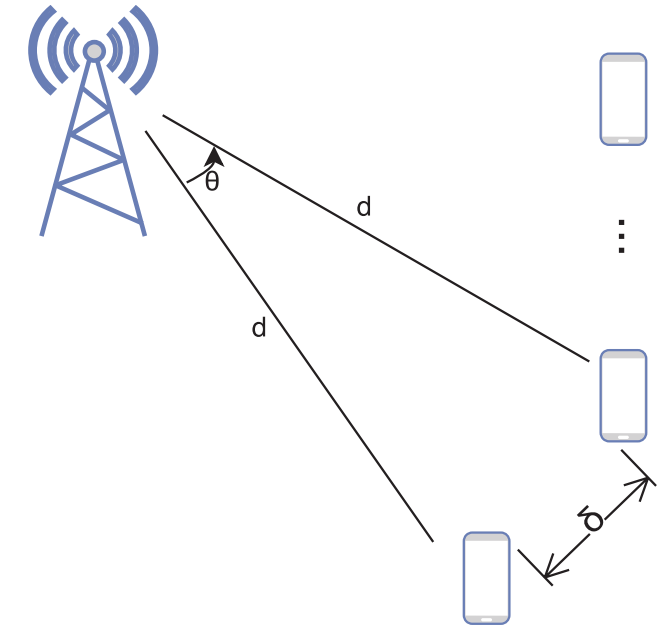

Fig. 1. System model illustration, highlighting the angle between users and both the inter-user path loss and UE-BS path losses.

is denoted with $\delta$. The system model is illustrated in Fig. 1. Using this model, the distance between users is $\delta=2 d \sin \left(\frac{\theta}{2}\right)$ and assuming a path loss exponent of $\gamma$, the path loss relation can be calculated as a function of $\theta$ with

$$
\frac{l_{d}}{l_{\delta}}=\frac{1 / d^{\gamma}}{1 / \delta^{\gamma}}=\left(\frac{\delta}{d}\right)^{\gamma}=\left(2 \sin \left(\frac{\theta}{2}\right)\right)^{\gamma} .
$$

Therefore, the relative path loss between users is given by $l_{\delta}=$ $\left(2 \sin \left(\frac{\theta}{2}\right)\right)^{-\gamma}$, as the average UE-BS path loss is normalized.

To consider PAPR, we are interested in the time domain transmitted signal. By denoting vectors collecting the persubcarrier transmit power allocations and symbols for stream $s$ as $\mathbf{p}_{s} \in \mathbb{R}^{N_{c} \times 1}$ and $\mathbf{x}_{s} \in \mathbb{C}^{N_{c} \times 1}$, and further stacking the transmit beamformers of all subcarriers accessing stream $s$ as $\tilde{\mathbf{V}}_{s}=\left[\begin{array}{llll}\mathbf{v}_{1, s} & \mathbf{v}_{2, s} & \ldots & \mathbf{v}_{N_{c}, s}\end{array}\right]^{\mathrm{T}} \in \mathbb{C}^{N_{c} \times K}$ we can express the time-domain transmitted signals from all users with the $N_{c} \times K$ matrix

$$
\mathbf{S}^{\text {data }}=\mathbf{F}^{-1} \sum_{s=1}^{K} \mathbf{P}_{s}^{\frac{1}{2}} \mathbf{X}_{s} \tilde{\mathbf{V}}_{s}
$$

where $\mathbf{P}_{s}=\operatorname{diag}\left(\mathbf{p}_{s}\right), \mathbf{X}_{s}=\operatorname{diag}\left(\mathbf{x}_{s}\right)$ and $\mathbf{F}$ is the discrete Fourier transform (DFT) matrix.

In this paper, we construct the transmitted signal using the Hughes-Hartogs bit and power allocation scheme following the implementation of [12]. The algorithm iteratively allocates bits on the subcarriers requiring the smallest power increment, given by

$$
\Delta p_{c, s}=\left(2^{\Delta b}-1\right) 2^{b_{c, s}} \frac{5}{8} \ln \left(\frac{1}{5 \mathrm{BER}_{c, s}}\right) \mathrm{G}_{c, s}^{-1},
$$

where $\Delta b$ denotes the number of increased bits, $b_{c, s}$ denotes the current bit allocation on stream $s$, subcarrier $c$ and $\mathrm{BER}_{c, s}$ is the subcarrier-wise bit error rate (BER) target. For simplicity, we consider a global BER target in this paper. The channel gain term $\mathrm{G}_{c, s}$ is dependent on the level of user cooperation 

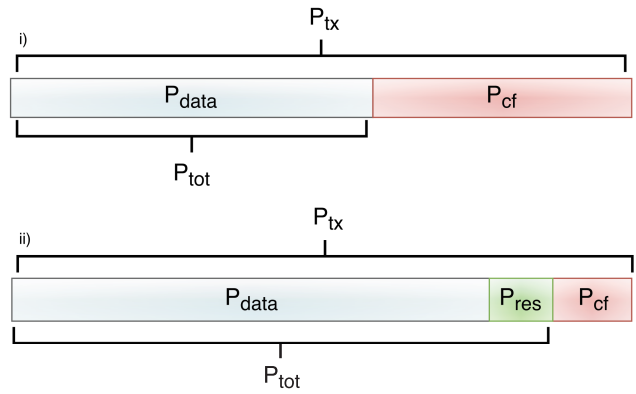

Fig. 2. Division of transmit power into its base components. i) represents the case where no nullspace beaming is applied, resulting in high PAPR. In ii), some of the total power pool $P_{\text {tot }}$ is allocated for nullspace beaming to reduce the PAPR.

and is given by

$$
\mathrm{G}_{c, s}=\frac{\Gamma_{c, s}}{N_{0} B},
$$

where $B$ is the subcarrier spacing and the term $\Gamma_{c, s}$ is either given by the eigenvalues, i.e., $\Gamma_{c, s}=\sigma_{c, s}^{2}$ or the zero forcing receiver, i.e., $\Gamma_{c, s}=\left\|\mathbf{u}_{c, s}\right\|_{2}^{-2}$ on stream $s$, subcarrier $c$. Furthermore, to enable user cooperation, the transmit data needs to be exchanged between users. The subcarrier-wise device-to-device power terms are calculated with [13]

$$
p_{c, s}^{\mathrm{UE}-\mathrm{UE}}=\frac{5}{8}\left(2^{b_{c, s}^{\mathrm{UE}-\mathrm{UE}}}-1\right) \ln \left(\frac{1}{5 \mathrm{BER}_{c, s}}\right) \frac{\left|l_{\delta}\right|^{2}}{N_{0} B},
$$

where $b_{c, s}^{\mathrm{UE}-\mathrm{UE}}$ is the average rounded integer number of transmitted bits on a subcarrier and $l_{\delta}$ is the path loss between users. The total power required for data exchange is the sum of these terms over $c, s$. Here, we assume that the data exchange between users happens on some off-band resource with same bandwidth as the UE-BS data transmission and is only dependent on the path loss between users. We also assume that the UE-BS channel is slow-varying enough so that the resource requirement for CSI exchange between users is negligible compared to data exchange.

Utilizing the power terms described above, the bit and power loading algorithm procures a sum-rate optimal bit and power allocation under a fixed power constraint $P_{\text {data }}$ while accounting for the data exchange between users. Let us define the total peak transmit power available (in $\mathrm{dB}$ ) as $P_{\mathrm{tx}}=P_{\text {data }}+P_{\text {res }}+P_{\mathrm{cf}}$, where $P_{\text {res }}$ is the reserve power factor that enables PAPR minimization and $P_{\mathrm{cf}}$ (cf, crest factor) is the transmit signal PAPR. Furthermore, we denote the total usable power pool as $P_{\text {tot }}=P_{\text {data }}+P_{\text {res }}$. The key idea is to allocate a part of the usable power pool for PAPR minimization in order to reduce $P_{\text {cf }}$. Thus, for a fixed peak transmit power budget $P_{\mathrm{tx}}$, we can increase the power allocated for data transmission, assuming that $P_{\text {res }}+P_{\text {cf }}$ is smaller than the original $P_{\mathrm{cf}}$ without PAPR minimization. As a result, we can execute a 2-dimensional search for a power division with respect to $P_{\text {data }}$ and $P_{\text {res }}$ that maximizes the system sum-rate. Another approach is to keep the average power budget fixed and only minimize the transmit signal PAPR. The division of the transmit power is further visualized in Fig. 2.

\section{PAPR Minimization Via Nullspace BEAMFORMING}

The main idea of nullspace beamforming is to utilize the unused space-frequency resources to transmit dummy symbols (complex coefficients) on the low gain dimensions orthogonal to the data-carrying ones. The dummy symbol carrying streams are in the receiver signal space if $M>K$ and can be ignored in data detection. The nullspace in question refers to the lowgain streams which are in the nullspace of the data-carrying high gain streams. The dummy symbols have an effect on the total transmitted time domain signal and, via optimization, the transmit signal PAPR can be significantly reduced. This can be done when there is full cooperation between users, i.e., collaborative beamforming.

The PAPR minimization problem is given by

$$
\begin{array}{ll}
\underset{\mathbf{M}}{\operatorname{minimize}} & P_{\mathrm{cf}}(\mathbf{M}) \\
\text { subject to } & \|\mathbf{M}\|_{\mathrm{F}}^{2} \leq P_{\text {res }} \\
& {[\mathbf{M}]_{c, s}[\mathbb{P}]_{c, s}=0, \forall c, s,}
\end{array}
$$

where $\mathbf{M}=\left[\begin{array}{llll}\mathbf{m}_{1} & \mathbf{m}_{2} & \ldots & \mathbf{m}_{K}\end{array}\right] \in \mathbb{C}^{N_{c} \times K}$ is the matrix containing the dummy symbols allocated for different subcarriers and $P_{\text {res }}$ denotes the portion of the total power pool reserved for PAPR minimization, i.e., $P_{\text {res }}=P_{\text {tot }}-P_{\text {data }}$. The matrix $\mathbb{P}=\left[\begin{array}{llll}\mathbf{p}_{1} & \mathbf{p}_{2} & \ldots & \mathbf{p}_{K}\end{array}\right] \in \mathbb{C}^{N_{c} \times K}$ contains the per-subcarrier transmit powers for data. The Frobenius norm constraint limits the power transmitted into the nullspace and the complementary slackness constraint ensures that dummy symbols are only placed on subcarriers containing no data symbols. The objective of (9) is the PAPR expression of the transmit signal, which is dependent on the dummy symbol allocation.

Our approach makes use of the result detailed in [9], where the authors propose that in order to reduce the transmit PAPR of the signal, only the peak transmit powers (i.e., the $l_{\infty}$-norm of the time domain transmit vector) need to be minimized. Following a similar model as with eq. (5), the total transmit signal in time domain can be formulated as

$$
\mathbf{S}^{\text {tot }}=\mathbf{F}^{-1} \sum_{s=1}^{K} \mathbf{P}_{s}^{\frac{1}{2}} \mathbf{X}_{s} \tilde{\mathbf{V}}_{s}+\mathbf{F}^{-1} \sum_{s=1}^{K} \operatorname{diag}\left(\mathbf{m}_{s}\right) \tilde{\mathbf{V}}_{s},
$$

where the vector $\mathbf{m}_{s}$ is the $s$ th column of matrix $\mathbf{M}$. Using the above time domain model, we can write the PAPR minimization problem in epigraph form by constraining the column-wise infinity norms of $\mathbf{S}^{\text {tot }}$. For a given time domain transmit sequence $\mathbf{S}^{\text {data }}$, the per-antenna signal peak minimization problem becomes

$$
\begin{array}{cl}
\underset{\mathbf{M}, \mathbf{t}}{\operatorname{minimize}} & \|\mathbf{t}\|_{2} \\
\text { subject to } & \left\|\left[\mathbf{S}^{\text {data }}\right]_{:, n}+\mathbf{F}^{-1} \sum_{s=1}^{K} \operatorname{diag}\left(\mathbf{m}_{s}\right)\left[\tilde{\mathbf{V}}_{s}\right]_{:, n}\right\|_{\infty} \leq t_{n}, \quad \forall n \\
& \|\mathbf{M}\|_{\mathrm{F}}^{2} \leq P_{\text {res }} \\
& {[\mathbf{M}]_{c, s}[\mathbb{P}]_{c, s}=0, \forall c, s .}
\end{array}
$$




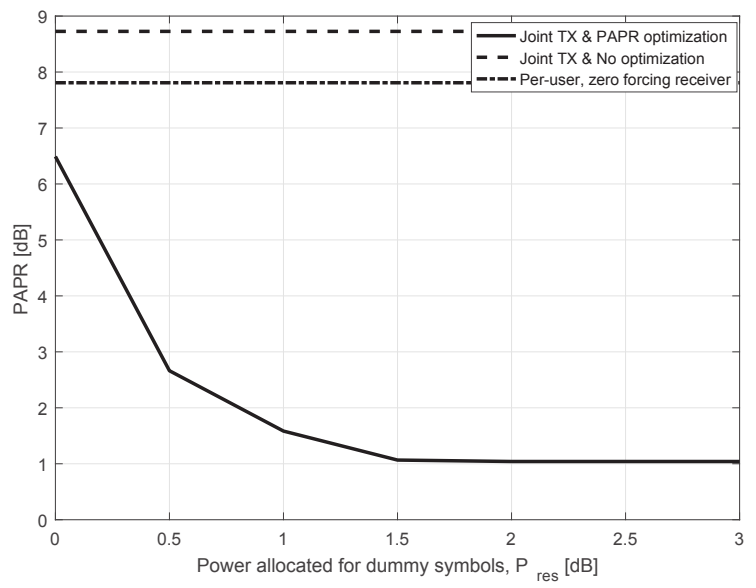

Fig. 3. Transmit PAPR as a function of the power reserved for PAPR minimization, $P_{\text {res }}$, for different levels of user cooperation.

Our approach to minimize the $l_{2}$-norm of the vector of peak constraints $\mathbf{t}=\left[\begin{array}{lll}t_{1} & t_{2} \ldots t_{K}\end{array}\right] \in \mathbb{R}^{K \times 1}$ is motivated by the fact that minimizing only the highest peak power (as was done in [9]) does not necessarily reduce the PAPR of antennas with lower mean transmit powers. Utilizing the $l_{2}$-norm prioritizes the higher antenna peaks in a quadratic sense, yet also allows the minimization of the lower peaks. ${ }^{1}$ The modified problem (11) is convex and can be optimally solved using interior point methods. However, there is a heuristic element involved in the choice of power allocation between data transmission and PAPR minimization, as $P_{\text {data }}$ and $P_{\text {res }}$ need to be obtained via a 2-dimensional search.

\section{NumericAl RESUltS}

This section details the performance simulations of our proposed PAPR minimization scheme. We considered a base station with $M=4$ transmit antennas serving $K=2$ singleantenna users in an OFDM system with $N_{c}=64$ subcarriers. The channel model had $L=40$ distinct paths per user, with 30 degree angular spread All the simulations were performed over 5000 symbol realizations (100 channel realizations with 50 symbols each). Our performance investigation was two-fold: first, we considered the throughput and PAPR performance as a function of the power reserved for PAPR minimization $P_{\text {res }}$ and second, we plotted the impact of inter-user distance on the system throughput.

In the PAPR and throughput simulations, the angle between users was set to $\theta=10^{\circ}$. The 95-percentile transmit PAPR was simulated as a function of $P_{\text {res }}$ with $P_{\text {data }}=10 \mathrm{~dB}$. We considered cases where users cooperated while utilizing the PAPR optimization and without, and also the non-cooperating case with zero forcing receiver for comparison. The results are illustrated in Fig. 3. From the figure it can be immediately

${ }^{1}$ Utilizing the $l_{\infty}$-norm instead would result in an approach similar to that of [9].

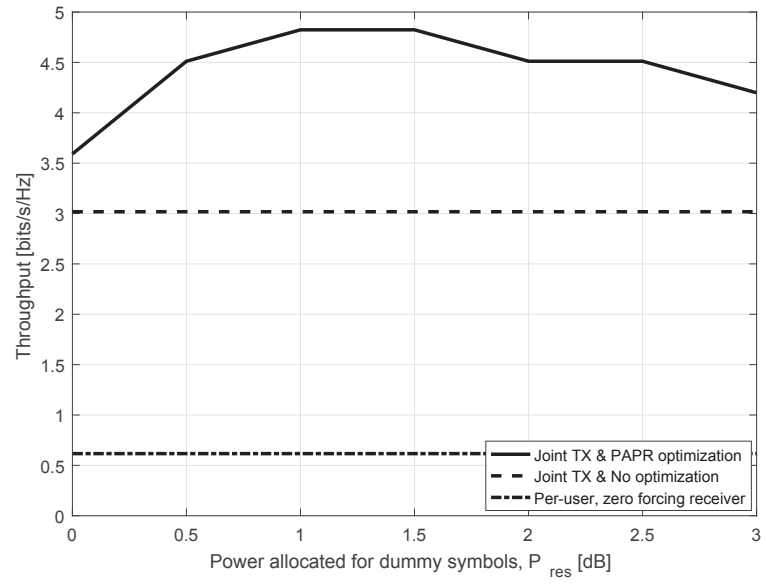

Fig. 4. System throughput as a function of the power reserved for PAPR minimization, $P_{\text {res }}$, for different levels of user cooperation.

seen that allocating $1-1.5 \mathrm{~dB}$ of the transmit power to PAPR minimization, the PAPR can be reduced from $9 \mathrm{~dB}$ to $1 \mathrm{~dB}$. On higher allocations the PAPR minimization no longer provides significant benefits and the scheme starts to consume the power allocated for data transmission. Interestingly, even at $P_{\text {res }}=0 \mathrm{~dB}$, the scheme can still provide reduced PAPR compared to the OFDM baseline. This is due to the nature of Hughes-Hartogs bit and power allocation which allocates power in chunks, rather than in a linear fashion. Under a strict data power constraint, this leaves some leftover power to be utilized for PAPR reduction. Furthermore, the non-cooperative zero forcing case has a slightly smaller PAPR than with joint transmission due to the lower SNR resulting in lower order modulation schemes.

The throughput performance was simulated with a constant peak transmit power constraint $P_{\mathrm{tx}}=15 \mathrm{~dB}$. To find the best combination of $P_{\text {data }}$ and $P_{\text {res }}$, a 2-dimensional search was conducted such that $P_{\text {data }}+P_{\text {res }}+P_{\mathrm{cf}}<P_{\mathrm{tx}}$. The simulation results are presented in Fig. 4. From the graph we can see that the system throughput can be significantly increased compared to the OFDM baseline by allocating a small amount of power to minimize the transmit PAPR. As with the PAPR simulations, allocating $1-1.5 \mathrm{~dB}$ of the transmit power for $P_{\text {res }}$ provides the highest throughput.

Finally, we investigated the effect of inter-user distance on the system throughput for different levels of user cooperation. The throughput was simulated as a function of the angle between users $\theta$ with a transmit peak power constraint of $P_{\mathrm{tx}}=15 \mathrm{~dB}$. The individual throughput points were obtained with the same 2-dimensional search as in the previous simulation. The effect of inter-user distance is illustrated in Fig. 5. The results indicate that with smaller inter-user angles the user cooperation with PAPR minimization dominates over the plain cooperative and non-cooperative cases. Then, as the angle gets larger, the joint transmission performance starts to degrade and 


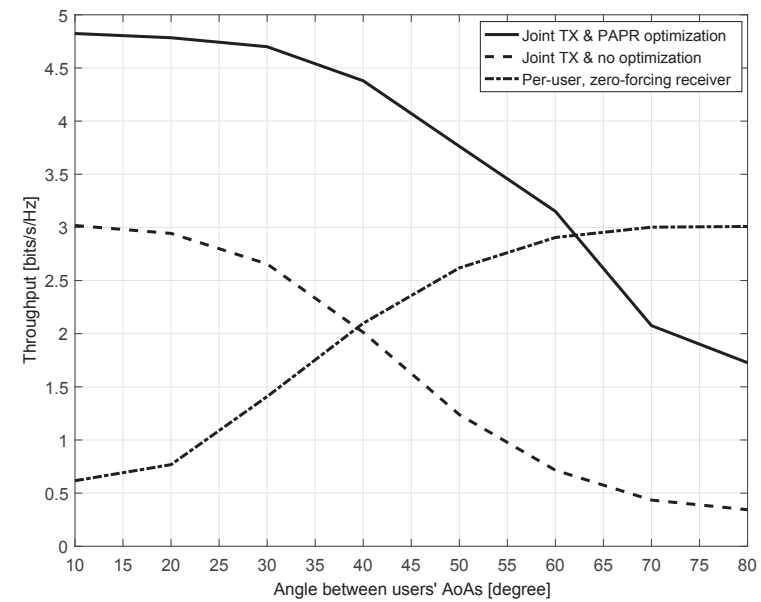

Fig. 5. Throughput performance as a function of the angle between users' AoAs, i.e., the inter-user distance.

the non-cooperative zero forcing case becomes a better option. This is due to the high amount of correlation in the user channels at small angles, which inhibits the separation capabilities of the zero forcing receiver. Then, as the angle gets larger, the correlation diminishes and zero forcing performs better. Utilizing the PAPR minimization scheme shifts the crossing point, where the non-cooperative case becomes preferable, towards larger inter-user angles. This is mainly due to the increase in data power provided by the PAPR minimization.

\section{Conclusions}

In this paper, we proposed a scheme that minimizes the transmit signal PAPR by exploiting the unused spacefrequency resources induced by joint data transmission. We also accounted for the power required to exchange the transmit data between users that is necessary for joint transmission to occur. Our proposed scheme is modular in the sense that to operate, it only requires knowledge of the transmitted time domain waveforms from all antennas, a portion of the usable power pool and some unused subcarriers. Therefore, any method of constructing the transmit signal and transmit beamformers are valid. The simulation results show that the transmit PAPR can be significantly reduced with only a small amount of extra allocated power. This enables the transmission of higher data rates compared to the OFDM baseline.

\section{REFERENCES}

[1] F. Peng and W. Ryan, "On the capacity of clipped OFDM channels," in Information Theory, 2006 IEEE International Symposium on, July 2006, pp. 1866-1870.

[2] R. Bauml, R. Fischer, and J. Huber, "Reducing the peak-to-average power ratio of multicarrier modulation by selected mapping," Electronics Letters, vol. 32, no. 22, pp. 2056-2057, Oct 1996.

[3] S. Muller and J. Huber, "OFDM with reduced peak-to-average power ratio by optimum combination of partial transmit sequences," Electronics Letters, vol. 33, no. 5, pp. 368-369, Feb 1997.
[4] R. F. H. Fischer and M. Hoch, "Directed selected mapping for peakto-average power ratio reduction in mimo ofdm," Electronics Letters, vol. 42, no. 22, pp. 1289-1290, Oct 2006

[5] B. S. Krongold and D. L. Jones, "Par reduction in ofdm via active constellation extension," IEEE Transactions on Broadcasting, vol. 49, no. 3, pp. 258-268, Sept 2003.

[6] A. Sendonaris, E. Erkip, and B. Aazhang, "User cooperation diversity. part i. system description," IEEE Transactions on Communications, vol. 51, no. 11, pp. 1927-1938, Nov 2003.

[7] — "Increasing uplink capacity via user cooperation diversity," in Information Theory, 1998. Proceedings. 1998 IEEE International Symposium on, Aug 1998, pp. 156-

[8] A. Asadi, Q. Wang, and V. Mancuso, "A survey on device-to-device communication in cellular networks," IEEE Communications Surveys Tutorials, vol. 16, no. 4, pp. 1801-1819, Fourthquarter 2014.

[9] C. Studer and E. G. Larsson, "Par-aware large-scale multi-user mimoofdm downlink," IEEE Journal on Selected Areas in Communications, vol. 31, no. 2, pp. 303-313, February 2013.

[10] D. Hughes-Hartogs, "Ensemble modem structure for imperfect transmission media," May 23 1989, uS Patent 4,833,706.

[11] A. F. Molisch, Wireless communications. John Wiley \& Sons, 2007.

[12] Q. Sun, L. Li, A. Tölli, M. Juntti, and J. Mao, "Optimal energy efficient bit and power loading for multicarrier systems," Communications Letters, IEEE, vol. 18, no. 7, pp. 1194-1197, July 2014.

[13] S. T. Chung and A. Goldsmith, "Degrees of freedom in adaptive modulation: a unified view," Communications, IEEE Transactions on, vol. 49, no. 9, pp. 1561-1571, Sep 2001. 